\title{
The Future of the Interaction between Industry, Universities, and Research Institutes
}

La Salle du Conseille à la Maison de l'Université de Haute Alsace on 29 November was the scene of the first EPS event to be organized at its new headquarters in Mulhouse, just over two months before the official transfer on 1 January 1997. The previous evening had seen the organization (jointly by EPS and the University) of a public lecture by Claude Weisbuch (Ecole Polytechnique de Palaiseau) on "Nanophysique". The meeting on the future interaction between industry, universities and research institutes was organized by Paul Phelan of University College, Dublin, on behalf of the EPS Interdivisional Group on Applied Physics and Physics in Industry. Twentyone representatives from Europe's industrial and academic research communities explored the problem in an interactive round-table discussion. Many of the participants were "boundary crossers"; having worked in both industrial and academic cultures. The key issues highlighted included:

- Complexity is a feature of the problem. Many paradoxes and differing views were revealed, precluding finding simple answers. A contingency approach may be needed to deal with differences between large and small companies, and countries.

- Industry wants external knowledge and expertise for applied research projects, and basic research at universities supported by governments. But relying on governments to support basic research was cautioned against by others. Above all, industry needs people for recruitment from universities.

- People issues featured strongly. Physics graduates are highly valued in a variety of industries, although university research training might benefit from some redesign; management courses may need to be included. People provide an effective technology transfer mechanism to industry, and spin-off companies, but the effectiveness of this transfer requires improvement; "interface expertise" is needed.

People may be more relevant than Intellec- tual Property in the commercialisation of physics.

EPS President Herwig Schopper opened the meeting by highlighting the importance of interacting with industry, and the need for mechanisms to encourage a fluid exchange of people. Science and technology are not important in the view of the West, and it is difficult to obtain funding for research in industry and universities. Meanwhile Japan regards current technology as mature, and is increasing research funding and looking to the universities for the technologies of the future.

Steward Bruyn described how a leading player in the telecommunications industry, like Nortel, increasingly needs to tap into external research knowledge it does not already have. Why does industry fund any university research? It is certainly not for charity. External research is not a cheap means for product development and is far from risk free. Industry invests in universities for pre-competitive research and early warning of potential breakthroughs; to help universities achieve academic excellence and provide highlyskilled people for industry; and for the potential leverage their support gives worldwide, particularly in developing countries. Olle Nilsson of Ericsson expressed similar views - the most important outcome from the relationship is educated people, although the international networks and research are also important. The influence of big industry must be balanced by other forces to promote renewal. Allan de Monchy (FOM and ex-Shell) believed that basic R\&D in universities should be encouraged by industry as an investment in the future, but it should be paid for by government. François Buchy of Thomson CSF proposed that there should be work between industry and universities to ensure that critical corporate technologies were understood.

Claude Weisbuch (Ecole Polytechnique, Palaiseau) warned that universities could not be the Bell Labs of the 9os', and that governments could not be relied on to support Europe's valuable university research resources. Kostas Glinos of the European Commission (DG3, Long-Term Research) suggested that human resources, rather than patents, are increasingly the key to industrial competitive advantage. The challenge is now to reconcile the different objectives (and cultures) of the academic and industrial communities. Business Innovation Centres were attempting to overcome the entrepreneurship lacking in some Universities.

Vincent McBrierty of Trinity College, Dublin - and formerly of Bell Labs stressed the need for physicists to have a holistic approach, covering technology, marketing and social implications. For example, although the technology behind the videophone was admirable, no-one wanted the product. Physicists and engineers must be trained in marketing, as many of those present agreed. Vincent $\mathrm{McBrierty}$ proposed knowledge as a new form of equity, and described the dynamic innovation focus of Trinity College, Dublin, with examples of successful industrial interaction, patent exploitation and research-driven start-up. "Culture Bridging" was succeeding in the universities of some smaller EU countries where SMEs are crucial. Markus Pessa, who has spun-off a company from his research in Tampere University, recommended that laboratories aim for ISO 9000 certification to attract industrial interaction. "How different the situation in different countries is" commented Fritz Schneider, former head of research at Daimler-Benz. Several university representatives were concerned about who would pay for the high cost of patenting. Much trading of patents takes place in industry; Nortel and Ericsson give one access to their patents. And in some cases the lead time and lifetime of a product is so short that there is little advantage in patenting. Concern was also expressed by several people over the lack of exclusivity and thus limited commercial exploita- 
tion that is often attached to governmentor EU-funded projects.

Claude Weisbuch (Ecole Polytechnique de Palaiseau) drew analogies between values in science and in product development: originality versus competitiveness, publishing versus patenting, breakthroughs versus incremental progress. However, his most far-reaching remarks related to the role of PhDs. On completing a $\mathrm{PhD}$, a person should be a problem-solving generalist rather than a narrow specialist, he said. Peter Melville of the Institute of Physics wondered whether this did not mean that a fundamental reappraisal of both undergraduate and postgraduate courses was required. Herwig
Schopper mentioned a particle physicist from CERN who got a job with Volkswagen on the basis that his $\mathrm{PhD}$ had demonstrated that he could work in a team, solve complex problems and work within time and budget. Although the movement of people is regarded as the most important aspect of the university-industry relationship, not all $\mathrm{PhDs}$ are as fortunate.

Clearly the EPS wants to improve the interface between industry, universities and research institutes. The importance of this initiative, and the appropriateness of the format was acknowledged by the participants. Ultimately the meeting succeeded in highlighting the complexity of the problem, and raising awareness. A good start has been made on the road which may be more of a journey than a destination, but before deciding what to do, the EPS needs to answer:

-What does industry want, and how do SMEs' needs differ from big industry?

- How do different countries deal with the problem?

- How can the interface management be improved?

- How can basic research in universities be adequately supported?

- Should university courses be modified and how?

Peter Melville (Institute of Physics)

\section{Use of Multimedia in Physics Teaching and Learning - Workshop 1996}

The use of PCs in university teaching (lectures, seminars, problem-solving, students' laboratories) is well accepted and organized nowadays: convincing software packages, physics textbooks in conjunction with discs, $\mathrm{PC}$ equipment for the teaching environment are readily accessible.

Recent developments in and the availability of multimedia - such as internet and web sites, information highways, CDROM, teleteaching via the net - combined with the declared intent of politicians (Taskforce Educational Software and Multimedia, European Commission 1995/1996 and Ministries for Education) that the physics community consider carefully whether multimedia are suitable for teach-ing and learning physics: this would con-stitute a logical progression of the PC in the direction of the multimedia.

Physicists interested in education therefore formed a group at the European level and organized the first Workshop 1996 in Munich, Germany (for further details, such as the list of participants, Workshop programme, and multimedia material, see the Workshop homepage below).

In a status report, current multimedia software was collected and evaluated; ideas and experience were exchanged. In subgroups, the participants started activites in the following areas:

1) Development of further multimedia material.
2) Teaching via internet, for example in-service physics teachers or off-campus physics students.

3) Pedagogical aspects such as testing, quality standards, evaluation, selflearning techniques.

4) Studio class approach, i.e. instead of lectures where students listen and watch passively, small groups in which they actively participate.

5) Adapting and experimenting with existing integrated pedagogical software.

Besides this multimedia workshop at the European level (more research oriented, future trends etc.), parallel national workshops were also established in each country (the aims are to make research products accessible for broader application, to consider national differences in the socio-cultural environment, and more.

There will be a Second European Workshop in Pavia, Italy ( 1997), organised by L.Borghi, as well as a Zweiter Arbeitskreis Multimedia in Berlin, Germany (March 1997), organised by R.Rass

\section{Information: H.J. Jodl, Fachbereich Physik, Universität Kaiserslautern, Deutschland Fax (49)-631-205-3677 \\ e-mail:jodl @ physik.uni-kl.de \\ http:// www . physik.uni-kl.de/w_jodl/w_jodl html}

\section{MPI for Polymer Research Mainz, Germany}

The Theory Department at the Max-Planck-Institute for Polymer Research has several openings for PhD-student positions. Research areas comprise different fields of Soft Matter Theory (Polymer Networks, Polyelectrolytes, Hydro Gels, Structure-PropertyRelation)

Potential topics contain numerical works, of theoretical-chemical and theoretical-physical character (PD Dr. Müller-Plathe, Dr. Dünweg, Prof. Kremer), as well as analyticaltheoretical investigations (Prof. Vilgis -statistical physics-, Prof. Pleiner-macroscopic theory-).

Besides a very good masters (or equivalent) degree in Physics, Chemistry or similar areas (for example: Material Sciences, Chemical Engineering, Mathematics) applicants should be willing to collaborate within the Theory Department as well as with experimental groups. Knowledge of Soft Matter Physics or Physical Chemistry is advantageous but not always required. We offer the usual PhD-students' grant, excellent working conditions, allowing for a swift completion of the studies ( 2.5 years).

Applications (containing resume, cv etc.) should be sent to the Director of the Theory Department, Prof. Dr. K. Kremer, Max-Planck-Institut für Polymerforschung, Postfach 3148, D-55021 Mainz; email: kremer@mpip-mainz.mpg,de 